\title{
Intravitreal Bevacizumab for Treatment of Diabetic Macular Edema
}

\author{
Jeong Won Seo, MD, In Won Park, MD \\ Department of Ophthalmology, Hallym University College of Medicine, Hallym University Sacred Heart Hospital, Anyang, Korea
}

\begin{abstract}
Purpose: To evaluate the effect of intravitreal bevacizumab on visual function and retinal thickness in patients with diabetic macular edema (DME).

Methods: Thirty eyes of twenty-eight patients (mean age, $57.9 \pm 13.8$ years) with DME were included in this study. Complete ophthalmic examination, including determination of best-corrected visual acuity (BCVA), stereoscopic biomicroscopy, and retinal thickness measurement by optical coherence tomography (OCT), was done at baseline and at each follow-up visit. All patients were treated with a $0.05 \mathrm{~mL}$ intravitreal injection containing $1.25 \mathrm{mg}$ of bevacizumab.

Results: All patients completed 3 months of follow-up with a mean follow-up period of $5.26 \pm 2.39$ months. The mean BCVA at baseline was $0.73 \pm 0.36$ logMAR, which significantly improved to $0.63 \pm 0.41(p=0.02), 0.58 \pm 0.36$ $(p=0.003)$, and $0.61 \pm 0.40 \operatorname{logMAR}(p=0.006)$ at 1 week, 1 month, and 3 months. Final BCVA analysis demonstrated that 15 eyes $(50 \%)$ remained stable and $12(40 \%)$ improved $\geq 2$ lines on BCVA. The mean central retinal thickness was $498.96 \pm 123.99 \mu \mathrm{m}$ at baseline and decreased to $359.06 \pm 105.97(p<0.001), 334.40 \pm 121.76$ $(p<0.001), 421.40 \pm 192.76 \mu \mathrm{m}(p=0.035)$ at 1 week, 1 month, and 3 months. No ocular toxicity or adverse effects were observed.

Conclusions: Intravitreal bevacizumab injection resulted in significant improvement in BCVA and central retinal thickness as early as 1 week after injection in patients with DME, and this beneficial effect persisted for up to 3 months. However, the slight reduction in this improvement at 3 months suggests that repeated bevacizumab injections might be necessary. To evaluate the long-term safety and efficacy, further prospective randomized controlled clinical trials will be needed.
\end{abstract}

Korean J Ophthalmol 2009;23:17-22 (c) 2009 by the Korean Ophthalmological Society.

Key Words: Diabetic macular edema, Intravitreal bevacizumab injection

Diabetic macular edema (DME) is the leading cause of visual loss in patients with diabetes mellitus, and it frequently leads to irreversible changes in visual acuity. ${ }^{1}$ DME is caused by excessive vascular permeability, which leads to leakage of fluid and plasma constituents, such as lipoproteins, into the retina. This then causes retinal thickening. The Early Treatment Diabetic Retinopathy Study (ETDRS) showed that focal laser photocoagulation is beneficial in the treatment of clinically significant macular edema, reducing the rate of moderate visual loss by $50 \%{ }^{2}$ However, only $3 \%$ of patients improved by $\geq 3$ lines of vision by the end of the study. Intravitreal triamcinolone acetonide (IVTA) injection has proven effective in improving vision and reducing macular thickness in DME,

Received: June 5, 2008 Accepted: November 18, 2008

Reprint requests to In Won Park, MD. Department of Ophthalmology, Hallym University Sacred Heart Hospital, \#896 Pyeongchon-dong, Dongangu, Anyang-si, Gyeonggi-do 431-070, Korea. Tel: 82-31-380-3835, Fax: 8231-380-3837, E-mail: piw@korea.com

* The author has no proprietary interest in any of the materials or equipment mentioned in this study. both as an initial treatment and as a second line therapy after unsuccessful laser therapy. ${ }^{3,4}$ However, its effect is temporary, and a number of side effects have been reported. ${ }^{5,6}$ Consequently, its therapeutic value remains unclear.

Vascular endothelial growth factor (VEGF) has been implicated as an important factor in the breakdown of the bloodretina barrier, with increased vascular permeability resulting in retinal edema in diabetic patients through affecting endothelial tight junction proteins. ${ }^{7}$ While the normal human retina contains VEGF, hypoxia stimulates the secretion of VEGF from retinal pigment epithelial cells. ${ }^{8,9}$ VEGF levels are significantly elevated in eyes with DME. ${ }^{10,11}$ In addition, VEGF concentrations are significantly higher in eyes with extensive macular leakage when compared to eyes with minimal leakage. ${ }^{11}$ Therefore anti-VEGF treatments have been proposed as an alternative adjunctive treatment for DME. ${ }^{12}$ Bevacizumab (Avastin, Genentech Inc., San Francisco, CA) is a complete full-length humanized antibody that binds to all subtypes of VEGF; it has been used successfully as a systemic drug in tumor therapy. ${ }^{13}$ Recent studies have demonstrated the usefulness of intravitreal injections of bevacizumab in the reduction of macular edema secondary to central retinal vein 
occlusion, vascular permeability, fibrovascular proliferation in retinal neovascularization secondary to proliferative diabetic retinopathy (PDR), and choroidal neovascularization secondary to age-related macular degeneration (AMD). ${ }^{14-17}$

The purpose of this retrospective study was to evaluate the effect of intravitreal bevacizumab on visual function and retinal thickness in patients with DME.

\section{Materials and Methods}

The present study was designed as a retrospective, consecutive case series study of eyes with DME treated with off-label intravitreal bevacizumab between March 2007 and February 2008. We reviewed the clinical records of 28 consecutive patients (30 eyes) with DME treated with at least one intravitreal injection of $1.25 \mathrm{mg}$ of bevacizumab. Because there are no evidence-based indications for the treatment of DME with bevacizumab, we included a wide range of patients with diffuse, clinically significant DME who did not respond to other treatments such as photocoagulation, intravitreal triamcinolone injection, or pars plana vitrectomy. The inclusion criteria for the study eye included (1) best-corrected visual acuity (BCVA) $\leq 20 / 40$, (2) clinically definite retinal thickening due to DME involving the center of the macula, (3) optical coherence tomography (OCT) central retinal thickness $\geq 275$ $\mu \mathrm{m}$, and (4) no history of treatment for DME within the prior 3 months. Exclusion criteria included macular edema due to a cause other than diabetes, other ocular condition that might affect macular edema or alter visual acuity, and evidence of external ocular infection.

Each patient underwent a complete eye examination, including determination of BCVA, slit-lamp examination, intraocular pressure (IOP) measurement, stereoscopic biomicroscopy of the retina using a 90-diopter lens, and retinal thickness measurement by OCT (Stratus OCT model 3000; Carl Zeiss Meditec Inc., San Leandro, CA), at baseline and at each visit. Patients were examined at 1 week and 1 month after injection, and then at 1- or 2-month intervals at the discretion of the investigator. OCT images were obtained by fast macular thickness map scan (6-radial line pattern), and the central retinal thickness was measured using retinal map analysis for the calculation of average thickness at the center point. Each patient's BCVA was transferred from his or her records and converted to a logarithm of the minimum angle of resolution (logMAR) scale for analysis.

Topical anesthesia was induced by applying proparacaine $(0.5 \%)$ eye drops before injection. The conjunctiva bulbi and the fornices were repeatedly rinsed with povidone-iodine, which was also applied to the eyelid margins and the lashes to avoid expression of the meibomian glands. After application of a sterile drape and subconjunctival injection of anesthesia ( $2 \%$ lidocaine containing 1:100,000 epinephrine), a 30-gauge needle on a $1 \mathrm{~cm}^{3}$ syringe was used to inject bevacizumab intravitreally through the pars plana 3.5 to $4.0 \mathrm{~mm}$ posterior to the limbus, at a dose of $1.25 \mathrm{mg}$ in $0.05 \mathrm{~mL}$. The needle was carefully removed using a sterile cotton applicator to prevent reflux. After injection, antibiotic eye drops were applied four times per day for 3 days.

All patients provided written informed consent, and they were informed of the off-label use of the drug and its potential risks and benefits, as well as the likelihood that additional treatments might be required. The paired $t$-test was used for comparison of preoperative and postoperative BCVA and central retinal thickness. The multiple regression analysis was performed to evaluate the associated factors influencing treatment success, and Mann-Whitney $U$ test was used to compare response to treatment between nonvitrectomized eyes and previously vitrectomized eyes. For all statistical tests, a $p$ value $<0.05$ was considered statistically significant. The data were analyzed using statistical software (SPSS, version 12.0, SPSS Inc, Chicago, Illinois, USA).

\section{Results}

Table 1. Baseline characteristics of the study eyes

\begin{tabular}{lcc}
\hline Variables & & $18: 12$ \\
\hline Gender, male:female & & $57.9 \pm 13.8$ \\
Age (years) & & $5: 25$ \\
Diabetes type 1:2 $\quad$ duration (years) & $15.9 \pm 7.6$ \\
Hypertension & $\quad$ focal laser treatment $^{\dagger}$ & 10 \\
Stage of retinopathy, severe NPDR* ${ }^{\dagger}$ & $12: 18$ \\
Preoperative treatment, & panretinal photocoagulation & 8 \\
& intravitreal injection of triamcinolone & 15 \\
& pars plana vitrectomy & 4 \\
Mean follow-up period (months) & & 6 \\
Baseline visual acuity (logMAR) & & $5.26 \pm 2.39$ \\
Baseline central retinal thickness $(\mu \mathrm{m})$ & $0.73 \pm 0.36$ \\
\hline
\end{tabular}

* NPDR $=$ noproliferative diabetic retinopathy; ${ }^{\dagger} \mathrm{PDR}=$ proliferative diabetic retinopathy. 


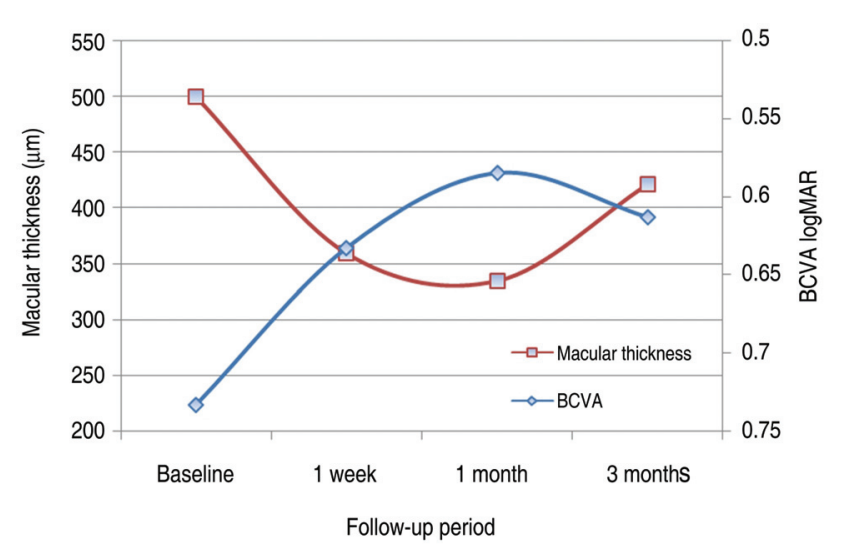

Fig. 1. Changes in best-corrected visual acuity (BCVA) and central retinal thickness measured by optical coherence tomography (OCT) after intravitreal bevacizumab injection.

Thirty eyes (28 patients) with a minimum of 3 months follow-up were included for analysis. The mean patient age was $57.9 \pm 13.8$ years, and $60 \%$ were male ( $18 \mathrm{men}, 12$ women). All patients completed 3 months of follow-up, with a mean follow-up period of 5.26 \pm 2.39 months (range, 3-11 months). Type 2 diabetes was present in $83.3 \%$ of patients, and type 1 diabetes was present in $16.7 \%$ of patients. Eighteen eyes $(60 \%)$ exhibited PDR, and twelve exhibited severe nonproliferative diabetic retinopathy (NPDR). Twenty-five eyes $(83.3 \%)$ had received at least one alternative therapy before intravitreal bevacizumab injection. Focal laser therapy had been applied once in 4 eyes and more than twice in 4 eyes. Full scatter panretinal laser therapy had been performed on 15 eyes (50\%), and 6 eyes (20\%) had undergone pars plana vitrectomy. Previous intravitreal injection of triamcinolone acetonide had been performed on 4 eyes at least 3 months before undergoing intravitreal bevacizumab injection. Additional baseline characteristics by treatment group are depicted in Table 1.

Improvements in visual acuity were noted from 1 week after intravitreal bevacizumab injection, and these statistically significant changes continued throughout the 3-month followup visit (Fig. 1). At baseline, the mean BCVA was $0.73 \pm 0.36$ $\operatorname{logMAR}$. This improved significantly to $0.63 \pm 0.41(p=0.02)$, $0.58 \pm 0.36(p=0.003)$, and $0.61 \pm 0.40 \log \operatorname{MAR}(p=0.006)$ at 1 week, 1 month, and 3 months, respectively. At 3-month follow-up, the BCVA was slightly decreased, but there was no significant difference in the mean BCVA between the 1and 3-month follow-up visits $(p=0.536)$. The visual acuity results are summarized in Figure 2. Final BCVA analysis by subgroup demonstrated that $15(50 \%)$ of 30 eyes remained stable, $12(40 \%)$ improved $\geq 2$ lines on BCVA, and $3(10 \%)$ deteriorated $\geq 2$ lines on BCVA (Table 2).

Mean central retinal thickness was $498.96 \pm 123.99 \mu \mathrm{m}$ (range, 275-733 $\mu \mathrm{m}$ ) at baseline by OCT. At 1 week postoperatively, the mean central retinal thickness measurement decreased to $359.06 \pm 105.97 \mu \mathrm{m}(p<0.001)$, and this significant improvement of retinal thickening continued through the 1 - and 3-month follow-up visits $(p<0.001$ and $p=0.035$,

Table 2. Best-corrected visual acuity (BCVA) analysis

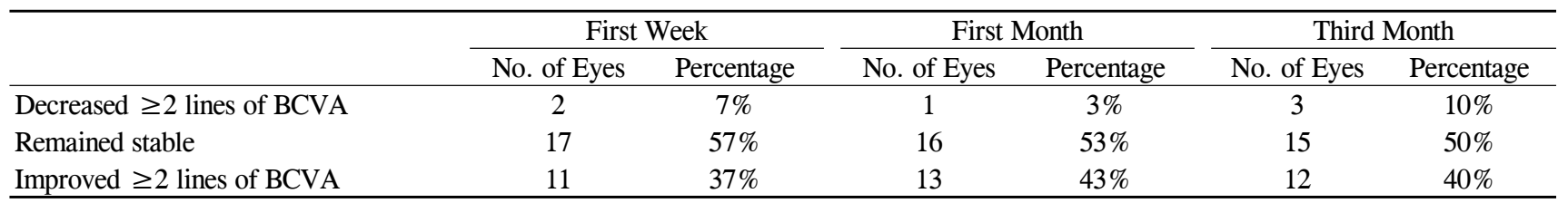
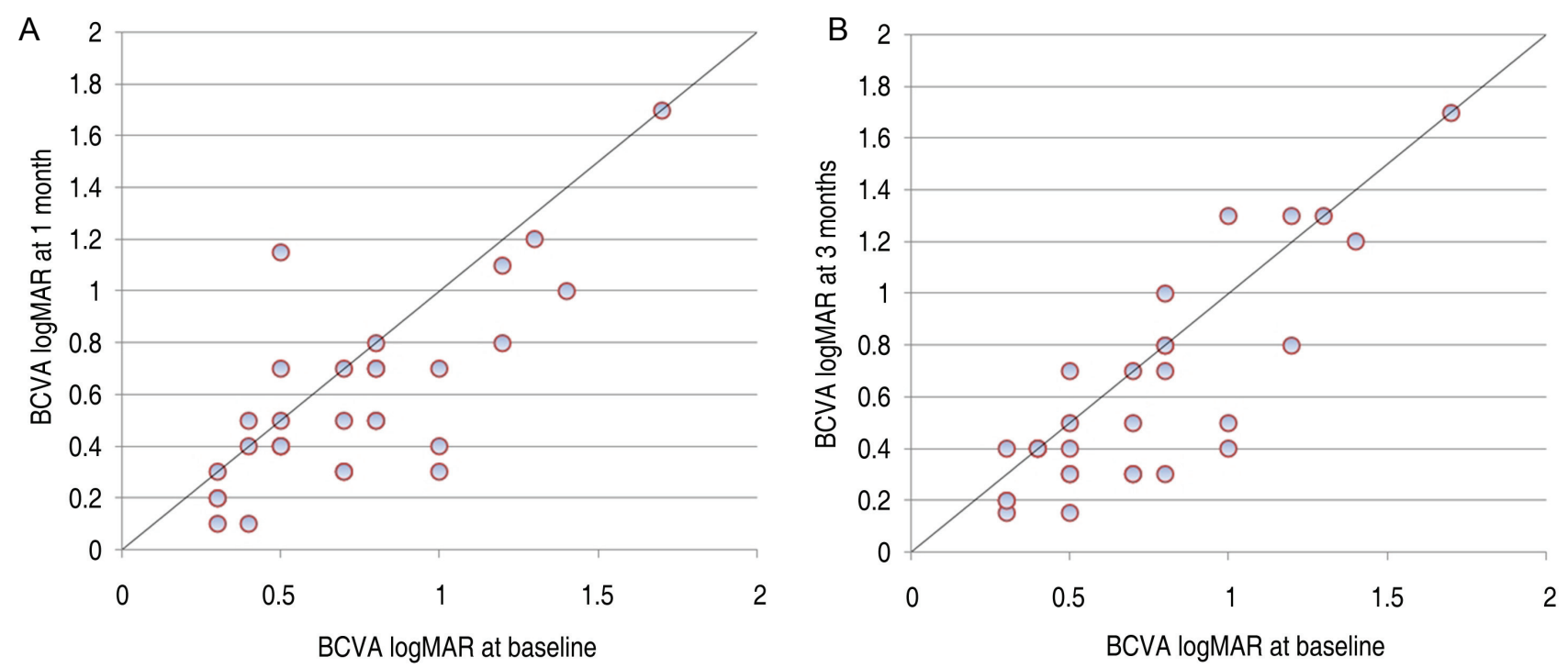

Fig. 2. Development of visual acuity (logMAR) evaluated after 1 month (A) and 3 months (B) of follow-up. 

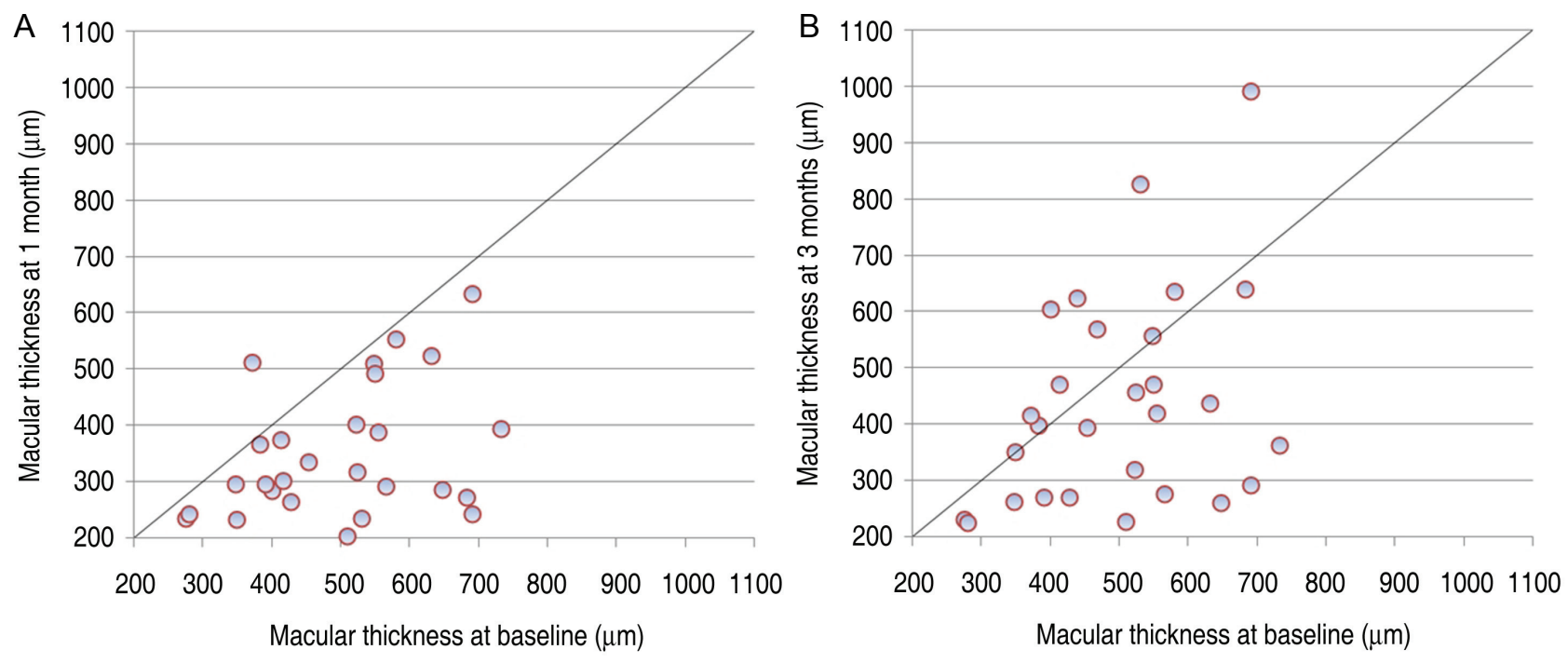

Fig. 3. Development of central retinal thickness measured by optical coherence tomography after 1 month (A) and 3 months (B) of follow-up.

respectively) (Fig. 1). However, at 3-month follow-up, mean central retinal thickness significantly increased to $421.40 \pm$ $192.76 \mu \mathrm{m}$, compared with 1-month follow-up (334.40土 $121.76 \mu \mathrm{m}, p=0.044)$. Figure 3 summarizes OCT-measured retinal thickness results.

Changes in visual acuity and changes in central retinal thickness did not vary substantially according to subject age ( $p=0.54$ and $p=0.95$, respectively), diabetic retinopathy severity ( $p=0.88$ and $p=0.14$ ), previous focal laser treatment ( $p=0.09$ and $p=0.76)$, previous panretinal photocoagulation $(p=0.31$ and $p=0.93$ ), or previous IVTA injection ( $p=0.79$ and $p=0.83$ ). There was a suggestion of greater effect on visual acuity in eyes that had not undergone pars plana vitrectomy compared with previously vitrectomized eyes $(p=0.025)$. The same effect was not observed concerning central retinal thickness $(p=0.98)$. Eyes with thicker retinas at baseline experienced a greater absolute and relative reduction in central retinal thickness at 1 month ( $p=0.001$ and $p=0.031$, respectively), but changes in visual acuity did not differ according to baseline visual acuity or baseline central retinal thickness $(p=0.06$ and $p=0.63$, respectively).

There were no cases of endophthalmitis, uveitis, IOP increase, or severe decrease in vision immediately after injection. At 3 months, no ocular or systemic adverse events were reported, including thromboembolic events (cerebrovascular accidents, transient ischemic attacks, myocardial infarctions, or peripheral vascular disease).

\section{Discussion}

DME is a manifestation of diabetic retinopathy that produces severe visual impairment. Although several treatment modalities are under investigation, the only demonstrated means to reduce the risk of vision loss from DME are laser photocoagulation, as demonstrated by the ETDRS ${ }^{2}$; intensive glycemic control, as demonstrated by the Diabetes Control and Complications Trial and the United Kingdom Prospective Diabetes Study; and blood pressure control, as demonstrated by the United Kingdom Prospective Diabetes Study. ${ }^{18,19}$ However, there has been interest in other treatment modalities, such as pharmacologic therapy with oral protein kinase $\mathrm{C}$ inhibitors and the use of intravitreal corticosteroids, because most laser-treated DME eyes do not exhibit satisfactory improvements in VA. ${ }^{20,21}$ Antibodies targeted to VEGF have also generated considerable interest and are being investigated.

VEGF is an endothelial cell-specific mitogen and angiogenic inducer in a variety of in vitro and in vivo models. ${ }^{22}$ It is upregulated by hypoxia, and it plays a role in DME and contributes to the excessive vascular permeability that leads to macular edema in diabetic patients. Bevacizumab is a full-length humanized monoclonal antibody that binds and inhibits all biologically active isoforms of VEGF. Although preclinical experimental data from primates suggested that the full-length antibody might not penetrate the internal limiting membrane of the retina, recent studies have shown full-thickness penetration of the retina within 24 hours. ${ }^{23,24}$ To our knowledge, all clinical and experimental studies presented thus far have not noted drug-related toxic effects in any retinal structure. ${ }^{14-17,25-30}$ Intravitreal injection of bevacizumab appears to have good efficacy in the treatment of wet AMD as a new treatment option. Injection of bevacizumab into the vitreous cavity, as is presently done mostly for patients with AMD, is based on the results of clinical reports clearly indicating an increase in visual acuity and a decrease in retinal thickness. ${ }^{15,16,27}$ In addition, other VEGF inhibitors, such as pegaptanib sodium (Macugen) - which binds to one VEGF isoform - have also been successfully used to treat DME in a published randomized, controlled, double-masked phase II multicenter trial. Subjects treated 
with pegaptanib had better visual acuity outcomes, were more likely to have reduction in central retinal thickness, and were less likely to need additional photocoagulation therapy at follow-up. ${ }^{12}$ In light of this information, intravitreal bevacizumab injection is expected to have good efficacy in the treatment of DME.

Recently, Haritoglou et al. ${ }^{28}$ published a prospective, noncomparative case series of patients with DME treated with $1.25 \mathrm{mg}$ bevacizumab. There was a significant reduction in macular thickness at 2 weeks $(p=0.002)$ and although mean visual acuity improved significantly at 6 weeks $(p=0.02)$, this was not sustained at 12 weeks. In the present investigation, however, we found that significant improvement in both visual acuity and retinal thickness was achieved soon after intravitreal bevacizumab injection, and the beneficial effects lasted for 3 months. The mean BCVA improved from $0.73 \pm 0.36 \log$ MAR at baseline to $0.63 \pm 0.41 \log$ MAR at $1-$ week follow-up $(p=0.02)$, and the mean central retinal thickness as measured by OCT also decreased significantly from $498.96 \pm 123.99 \mu \mathrm{m}$ at baseline to $359.06 \pm 105.97 \mu \mathrm{m}$ at 1-week follow-up $(p<0.001)$. At 1 month after the injection of bevacizumab, this beneficial effect on BCVA and central retinal thickness appeared to be most prominent in the current study. Thirteen (43\%) of 30 eyes showed an improvement in BCVA by 2 or more lines, and only 1 eye $(3 \%)$ decreased $\geq 2$ lines on BCVA at 1-month follow-up. In addition, the central retinal thickness showed a considerable reduction (33\%): from $498.96 \pm 123.99 \mu \mathrm{m}$ at baseline to $334.40 \pm 121.76 \mu \mathrm{m}$ at 1 month. Although the duration of action of intravitreal bevacizumab is unknown, recent electrophysiologic and retinal penetration studies have reported that full thickness retinal penetration is present at 24 hours. ${ }^{24}$ This may explain the earlier clinical effects of intravitreal bevacizumab observed in the current study.

A recent report from the Pan-American Collaborative Retina Study Group showed a significant decrease of DME one month after intravitreal injection of bevacizumab, and those decreased levels were kept for up to six months. ${ }^{29}$ In our study, the reduction of central retinal thickness and the improvement of BCVA were observed at 1 week, and these results were maintained for up to 3 months. However, at 3-month follow-up, a slight decrease in visual acuity and an increase in retinal thickness were observed as compared with the 1-month follow-up. The central retinal thickness increased significantly from $334.40 \pm 121.76 \mu \mathrm{m}$ at 1 month to $421.40 \pm$ $192.76 \mu \mathrm{m}$ at 3 months $(p=0.044)$. This slight reduction in the improvement of visual acuity and central retinal thickness at 3-month follow-up suggests that repeated intravitreal bevacizumab injections may be necessary within 3 months to maintain a beneficial effect. In this study, 6 (20\%) of 30 eyes received the second injection at a mean of 3.8 months. Re-treatment was done only in cases of BCVA deterioration and increased macular edema on OCT. It is possible that a different dosing schedule, such as a series of injections every 3 months for an extended period, may be superior to the method used in this study. However, we chose to re-treat the recurrent cases only, because data concerning toxicity and duration of action of intravitreal bevacizumab were not sufficient at the beginning of the study.

The breakdown of endothelial tight junctions and loss of the blood-retina barrier that lead to DME can be associated with both nonproliferative diabetic retinopathy and PDR. The present study demonstrates a comparable population of PDR and NPDR patients with macular edema. The results of the present study indicate that intravitreal bevacizumab injections may have a beneficial effect on retinal thickness and visual acuity, independent of the type of diabetic retinopathy. In addition, previous treatments, such as focal laser treatment, panretinal photocoagulation, or IVTA injection, did not influence the results of the study, except in the case of previous vitrectomy. Previously vitrectomized eyes showed no increase in visual acuity, and this finding is consistent with a previous study that found there was no change in BCVA or foveal thickness after intravitreal bevacizumab injection for DME in previously vitrectomized eyes. ${ }^{30}$ This outcome may be attributable to rapid clearance of intravitreal bevacizumab and insufficient sustained therapeutic levels in vitrectomized eyes. Further studies are warranted due to the relatively small number of participants in this study.

In conclusion, intravitreal bevacizumab injection appears to result in significant improvement in BCVA and reduction in central retinal thickness as early as 1 week after injection, and this beneficial effect was shown to persist for up to 3 months. However, the slight reduction in improvement in visual acuity and central retinal thickness at 3-month followup suggests that repeated bevacizumab injections might be necessary within 3 months to maintain its effect, as the drug is well tolerated and there are no safety concerns. To evaluate the long-term safety and efficacy of this new treatment, further prospective randomized controlled clinical trials will be needed, with scheduled re-injection and longer follow-up.

\section{References}

1. Ferris FL 3rd, Patz A. Macular edema: a complication of diabetic retinopathy. Surv Ophthalmol 1984;28:452-61.

2. Photocoagulation for diabetic macular edema. Early Treatment Diabetic Retinopathy Study Report Number 1. Early Treatment Diabetic Retinopathy Study Research Group. Arch Ophthalmol 1985; 103:1796-806.

3. Jonas JB, Kreissig I, Sofker A, Degenring RF. Intravitreal injection of triamcinolone for diffuse diabetic macular edema. Arch Ophthalmol 2003;121:57-61.

4. Martidis A, Duker JS, Greenberg PB, et al. Intravitreal triamcinolone for refractory diabetic macular edema. Am J Ophthalmol 2002;109:920-7.

5. Jonas JB, Kreissig I, Degenring RF. Intraocular pressure after intravitreal injection of triamcinolone acetonide. Br J Ophthalmol 2003;87:24-7.

6. Patelli F, Fasolino G, Radice P, et al. Time course of changes in retinal thickness and visual acuity after intravitreal triamcinolone acetonide for diffuse diabetic macular edema with and without previous macular laser treatment. Retina 2005;26:840-5. 
7. Qaum T, Xu Q, Joussen AM, et al. VEGF-initiated blood-retinal barrier breakdown in early diabetes. Invest Ophthalmol Vis Sci 2001;42:2408-13.

8. Aiello LP, Avery RL, Arrigg PG, et al. Vascular endothelial growth factor in ocular fluid of patients with diabetic retinopathy and other retinal disorders. N Engl J Med 1994;331:1480-7.

9. Adamis AP, Miller JW, Bernal MT, et al. Increased vascular endothelial growth factor levels in the vitreous of eyes with proliferative diabetic retinopathy. Am J Ophthalmol 1994;118: 445-50.

10. Funatsu H, Yamashita $H$, Noma $H$, et al. Increased levels of vascular endothelial growth factor and interleukin-6 in the aqueous humor of diabetics with macular edema. $\mathrm{Am} \mathrm{J}$ Ophthalmol 2002;133:70-7.

11. Funatsu H, Yamashita H, Ikeda T, et al. Angiotensin II and vascular endothelial growth factor in the vitreous fluid of patients with diabetic macular edema and other retinal disorders. Am J Ophthalmol 2002;133:537-43.

12. Cunningham ET Jr, Adamis AP, Altaweel M, et al. A phase II randomized double-masked trial of pegaptanib, an anti-vascular endothelial growth factor aptamer, for diabetic macular edema. Ophthalmology 2005;112:1747-57.

13. Ferrara N, Hillan KJ, Gerber HP, Novotny W. Discovery and development of bevacizumab, an anti-VEGF antibody for treating cancer. Nat Rev Drug Discov 2004;3:391-400.

14. Spaide RF, Fisher YL. Intravitreal bevacizumab (Avastin) treatment of proliferative diabetic retinopathy complicated by vitreous hemorrhage. Retina 2006;26:275-8.

15. Michels S, Rosenfeld PJ, Puliafito CA, et al. Systemic bevacizumab (Avastin) therapy for neovascular age-related macular degeneration. Twelve-week results of an uncontrolled openlabel clinical study. Ophthalmology 2005;112:1035-47.

16. Avery RL, Pieramici DJ, Rabena MD, et al. Intravitreal bevacizumab (Avastin) for neovascular age-related macular degeneration. Ophthalmology 2006;113:363-72.

17. Iturralde D, Spaide RF, Meyerle CB, et al. Intravitreal bevacizumab (Avastin) treatment of macular edema in central retinal vein occlusion: a short-term study. Retina 2006;26:279-84.

18. The effect of intensive treatment of diabetes on the development and progression of long-term complications in insulindependent diabetes mellitus. Diabetes Control and Complications Trial Research Group. N Engl J Med 1993;329:977-86.

19. Tight blood pressure control and risk of macrovascular and microvascular complications in type 2 diabetes: UKPDS 38. UK Prospective Diabetes Study Group. BMJ 1998;317:703-13.

20. Strom C, Sander B, Klemp K, et al. Effect of ruboxistaurin on blood-retinal barrier permeability in relation to severity of leakage in diabetic macular edema. Invest Ophthalmol Vis Sci 2005;46:3855-8.

21. Campochiaro PA, C99-PKC412-003 Study Group. Reduction of diabetic macular edema by oral administration of the kinase inhibitor PKC412. Invest Ophthalmol Vis Sci 2004;45:922-31.

22. Ferrara N. Vascular endothelial growth factor: basic science and clinical progress. Endocr Rev 2004;25:581-611.

23. Mordenti J, Thomsen K, Licko V, et al. Intraocular pharmacokinetics and safety of a humanized monoclonal antibody in rabbits after intravitreal administration of a solution or a PLGA microsphere formulation. Toxicol Sci 1999;52:101-6.

24. Shahar JS, Avry RL, Heilweil G, et al. Electrophysiologic and retinal penetration studies following intravitreal injection of bevacizumab. Retina 2006;26:262-9.

25. Maturi RK, Bleau LA,Wilson DL. Electrophysiologic findings after intravitreal bevacizumab (Avastin) treatment. Retina 2006; 26:270-4

26. Manzano RP, Peyman GA, Khan P, Kivilcim M. Testing intravitreal toxicity of bevacizumab (Avastin). Retina 2006;26: 257-61.

27. Rosenfeld PJ, Moshfeghi AA, Puliafito CA. Optical coherence tomography findings after an intravitreal injection of bevacizumab (Avastin) for neovascular age-related macular degeneration. Ophthalmic Surg Lasers Imaging 2005;36:331-5.

28. Haritoglou C, Kook D, Neubauer A, et al. Intravitreal bevacizumab (Avastin) therapy for persistent diffuse diabetic macular edema. Retina 2006;26:999-1005.

29. Arevalo JF, Fromow-Guerra J, Quiroz-Mercado H, et al. Primary intravitreal bevacizumab (Avastin) for diabetic macular edema. Ophthalmology 2007;114:743-50.

30. Yanyali A, Aytug B, Horozoglu F, Nohutcu AF. Bevacizumab (Avastin) for diabetic macular edema in previously vitrectomized eyes. Am J Ophthalmol 2007;144:124-6. 